\title{
A Microdischarge-Based Monolithic Pressure Sensor
}

\author{
Christine Kay Eun, Xin Luo, Jun-Chieh Wang, Zhongmin Xiong, Mark Kushner, and \\ Yogesh Gianchandani, Fellow, IEEE
}

\begin{abstract}
This paper describes the investigation of a microdischarge-based approach for sensing the diaphragm deflection in a monolithically fabricated pressure sensor. This transduction approach is appealing from the viewpoint of miniaturization. The device consists of a deflecting $\mathrm{Si}$ diaphragm with a sensing cathode, and a glass substrate with an anode and a reference cathode. The total exterior volume of the device is $0.05 \mathrm{~mm}^{3}$; typical electrode size and separations are 35 and $10 \mu \mathrm{m}$. Pulsed microdischarges are initiated in a sealed chamber formed between $\mathrm{Si}$ and glass chips, and are filled with Ar gas. External pressure deflects the Si diaphragm and changes the interelectrode spacing, thereby redistributing the current between the anode and two competing cathodes. The differential current is indicative of the diaphragm deflection which is determined by the external pressure. A 6-mask microfabrication process is investigated for device fabrication. Electrode connections to the interior of the chamber are provided by laser drilling and copper electroplating through high aspect ratio glass vias. The $\mathrm{Si}$ and glass substrates are bonded by $\mathrm{Au}-\mathrm{In}$ eutectic. The redistribution of plasma current between competing cathodes, as a consequence of diaphragm deflection over a range of pressure, was experimentally demonstrated. First principles modeling of transient microdischarges have provided insights to the fundamental processes responsible for the differential current and guidance for scaling the device to smaller dimension.

[2013-0200]
\end{abstract}

Index Terms-Through-glass vias (TGVs), electroplating, eutectic bonding, plasma modeling.

\section{INTRODUCTION}

A VARIETY of microscale pressure sensing solutions have been explored in the past five decades, of which the

Manuscript received June 21, 2013; revised February 6, 2014; accepted March 6, 2014. Date of publication March 28, 2014; date of current version November 25, 2014. This work was supported by the Advanced Energy Consortium (AEC). Subject Editor A. Seshia.

C. K. Eun is with the Center for Wireless Integrated MicroSensing and Systems, University of Michigan, Ann Arbor, MI 48109 USA; and also with the Department of Electrical Engineering and Computer Science, University of Michigan, Ann Arbor, MI 48109 USA (e-mail: eunc@umich.edu).

$\mathrm{X}$. Luo is with the Center for Wireless Integrated MicroSensing and Systems, University of Michigan, Ann Arbor, MI 48109 USA; and also with the Department of Mechanical Engineering, University of Michigan, Ann Arbor, MI 48109 USA (e-mail: xinluo@umich.edu).

J.-C. Wang, Z. Xiong, and M. Kushner are with the Department of Electrical Engineering and Computer Science, University of Michigan, Ann Arbor, MI 48109 USA (e-mail: junchwan@umich.edu; zxiong@umich.edu; mjkush@umich.edu).

Y. Gianchandani is with the Center for Wireless Integrated MicroSensing and Systems, University of Michigan, Ann Arbor, MI 48109 USA; the Department of Electrical Engineering and Computer Science, University of Michigan, Ann Arbor, MI 48109 USA; and also with the Department of Mechanical Engineering, University of Michigan, Ann Arbor, MI 48109 USA (e-mail: yogesh@umich.edu).

Color versions of one or more of the figures in this paper are available online at http://ieeexplore.ieee.org.

Digital Object Identifier 10.1109/JMEMS.2014.2312174 most commonly used are piezoresistive and capacitive pressure sensors [1]. Piezoresistive sensors typically measure stress in a diaphragm as it deflects in response to pressure. In contrast, capacitive pressure sensors respond to diaphragm deflection rather than stress. The smallest micromachined pressure sensors that have been reported - e.g., for use within cardiac catheters - use these transduction techniques [1]. For both types of sensors, the side-dimensions of the diaphragms of the smallest devices have been about $1 \mathrm{~mm}$ long. Further reduction in size has been a challenge for both approaches, but for different reasons.

For piezoresistive pressure sensors, reducing the diaphragm diameter presents a challenge in localizing the resistor. If the resistor extends too far from the edge toward the center of the diaphragm, it loses signal due to stress averaging: the stress on the upper surface of the diaphragm changes from tensile at the perimeter to compressive at the center, with a null point located in between. Making the resistor smaller is a challenge as well. Smaller resistors demand more current to generate a measurable voltage, and are relatively imprecise, which affects calibration and yield. Resistors inherently have a high temperature sensitivity, which makes this transduction approach less appealing for high temperature applications. Piezoresistive sensors do have relatively low output impedance, which means that the sensing circuit does not have to be located in the immediate proximity of the sensor. The equivalent noise pressure from piezoresistive pressure sensors increases as $1 / r^{4}$, where $r$ is the equivalent radius of the diaphragm [1].

Capacitive pressure sensors present a scaling challenge because the capacitance decreases in proportion to the area of the diaphragm. This scaling puts the burden of detection on the interface circuit, which must not only be precise, but must also be located in the immediate vicinity of the sensor in order to prevent the signal - which comes from a high impedance output, and hence is inherently weak - from leaking into parasitic capacitance. Another consequence of the reduced capacitance is the increase in $\mathrm{k}_{\mathrm{B}} \mathrm{T} / \mathrm{C}$ thermal noise. Together with other noise sources, the equivalent noise pressure from capacitive pressure sensors increases as $1 / r^{5}$ [1]. While capacitive pressure sensors have about $10 \times$ lower sensitivity to temperature than piezoresistive devices, the proximal interface circuit must be tolerant of high temperature environments as well.

Recently, an approach to pressure control and sensing based on microdischarges has been reported [2]-[4] Microdischarges are localized glow discharge plasmas or arcs created in gaseous media, which, due to their size, have characteristics different from larger scale discharges [5]. Microdischarges can be used 
in a variety of microsensors, including micro total analysis systems that use optical emission spectroscopy for chemical sensing [6]-[8], radiation detectors, sputter ion pumps, etc. [9]. Pressure and vacuum sensors based on this method of transduction utilize the change in plasma distribution within a cavity that may be caused either by a change in the plasma pressure [3] or by the deflection of a plasma electrode under external pressure [4]. Sensitivity levels in the range of 40,000-75,000 $\mathrm{ppm} / \mathrm{kPa}$ have been reported [3], which are comparable to piezoresistive pressure sensors. However, as in capacitive pressure sensors, linearity is compromised. The inherent signal levels are large compared to both capacitive and piezoresistive devices, eliminating the need for a proximal interface circuit - and, in fact, substantially reducing the need for amplification. This is very appealing from the viewpoint of miniaturization. Microdischarge cells that have been reported to date are at least $100 \times$ smaller in area than the smallest capacitive and piezoresistive pressure sensors that have been reported. For example, the discharge gap in cells used for plasma display panels can be less than $100 \mu \mathrm{m}$ [1]. Microdischarge-based displays with $50 \times 50 \mu \mathrm{m}^{2}$ cell size have been reported [11]. Emission spectroscopy of microdischarges for portable chemical sensing of liquids and gases has been reported for $50 \mu \mathrm{m}$ gaps between planar metal features [12]. A constraint is that microdischarge-based pressure sensors need high voltage to initiate discharges in the operation.

This paper ${ }^{1}$ describes a microdischarge-based approach for the measurement of diaphragm displacements in a monolithically fabricated device. The exterior volume of the device is only $0.05 \mathrm{~mm}^{3}$, which is $\approx 30 \times$ smaller than prior work [4]. The design of the structure is described in Section II, followed by microplasma modeling in Section III. Fabrication processes are addressed in Section IV. Preliminary experiments and results are provided in Section V.

\section{Device Concept And Design}

\section{A. Device Concept}

The device primarily consists of a glass substrate with copper filled through-glass vias (TGVs), a silicon diaphragm, one anode, and two competing cathodes, as shown in Fig. 1. A microdischarge chamber is formed by the glass substrate, silicon diaphragm, and a Au-In eutectic bond ring. All three electrodes are made of thin-film Ni. The anode $(A)$ and reference cathode $(K I)$ are located on the glass side facing the microdischarge chamber. The sensing cathode $(K 2)$ is located on the silicon diaphragm, and is electrically connected to the exterior contact pad through a doped silicon layer and the $K 2$ contact, which is a sandwich of Au and In layers in the interior of the chamber that mates with a TGV. All the electrical connections from within the chamber are routed to the exterior of the glass substrate through copper filled TGVs. An anode electrode placed in the center of the diaphragm would have provided the highest electrode displacement. However, given the diaphragm area available and the location of reference cathode $K 1$, the anode electrode $A$ is offset from the center.

\footnotetext{
${ }^{1}$ Portions of this paper appear in conference abstract form in [13].
}

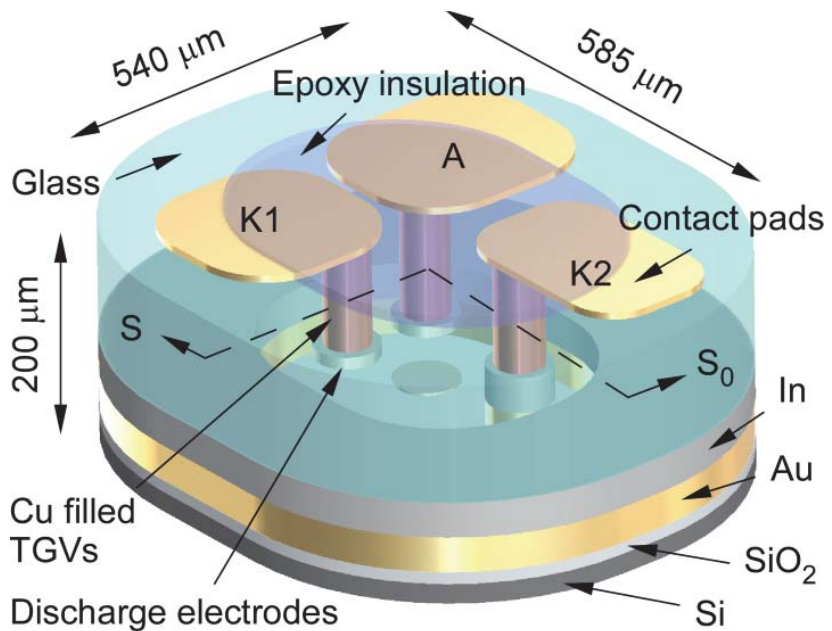

(a)



(b)

Fig. 1. Concept of the microdischarge based pressure sensor. (a) 3D model of the pressure sensor. (b) $\mathrm{S}-\mathrm{S}_{0}$ view of the structure. $I 1$ and $I 2$ are discharge currents from two discharge paths.

In this three-electrode configuration, a voltage pulse is applied to initiate microdischarges, and two current paths are established between the anode and the two cathodes. (The pulsed nature of the microdischarge reduces power consumption and parasitic heating, but requires customized code for simulations and modeling, as discussed later.) As the diaphragm deflects due to external pressure, the spacing between the anode and the sensing cathode $(A K 2)$ decreases, but the spacing between the anode and the reference cathode $(A K l)$ is essentially unaffected. This change of interelectrode spacing redistributes the spatial current: the ratio of sensing current (denoted by I2) between $A K 2$, and reference current (denoted by $I 1$ ) between $A K 1$. The differential current, expressed as a fraction of the total peak current $(I I-I 2) /(I I+I 2)$, can then be used as the sensor output to indicate the value of external pressure. By using this relative change in current, the absolute current becomes less important, which minimizes the consequence of pulse-to-pulse variation in microdischarge characteristics.

\section{B. Structural Design}

The diaphragm is made of silicon to enable both large diaphragm deflection, within the fracture limit, and electrical conductivity. The eutectic bond ring is $200 \mu \mathrm{m}$-wide; it forms a sealed chamber (185 $\mu$ m-long, $140 \mu$ m-wide) and determines the chamber height (and consequently the $A K 2$ interelectrode spacing). With this approach, etching the 


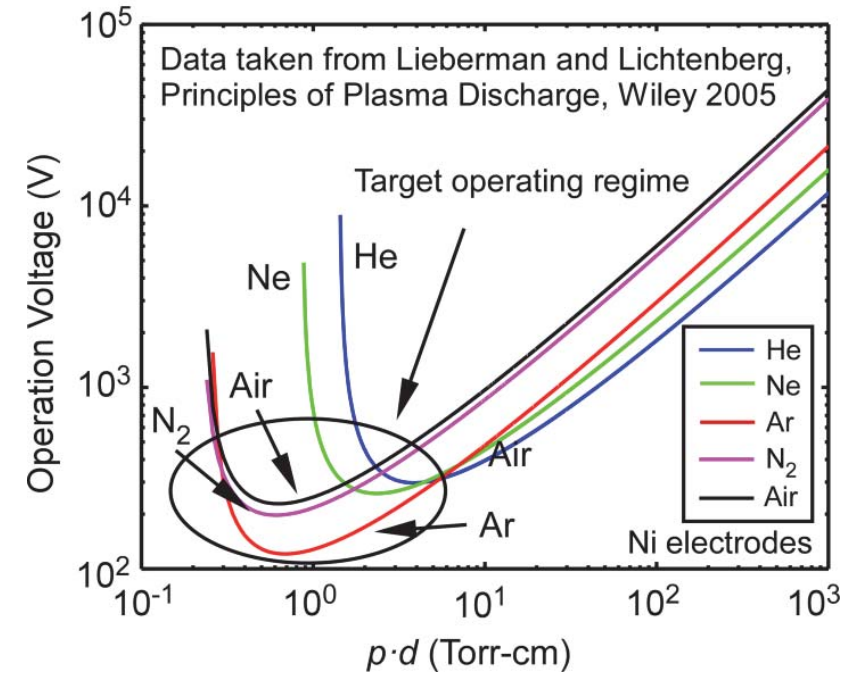

Fig. 2. Paschen curves for various fill-gases, which illustrate the relationship between operating voltage and $p \cdot d$ product, where $p$ is the pressure and $d$ is the interelectrode spacing. Discharge electrodes are Ni. The original data in this plot were reported in [20].

silicon or the glass to form a chamber is avoided. Electrostatic finite element analysis (FEA) using $\mathrm{COMSOL}^{\circledR}$ confirms that the use of this conductive bond ring as a spacing layer has little or no impact on the electric field profile. The discharge electrodes are made of nickel, which offers several benefits, including a high secondary electron emission coefficient (that contributes to a lower operation voltage), a high resistance to oxidation compared to alternatives [4], a convenient thin film deposition or electroplating, and ease of patterning. The architecture is designed with the vias in a glass wafer to provide adequate electrical isolation between the electrodes during device operation. This isolation allows the use of operating voltages that are $300-500 \mathrm{~V}$ or even higher. These vias are filled by copper electroplating. Prior work on 3D interconnect for integrated circuits (ICs) has mainly focused on through-silicon vias [14]-[17] for low-voltage devices. Through-glass vias have been investigated as means for providing better insulation and low-cost 3D packaging of ICs [18], [19]. More details of our approach to TGV fabrication are described in Section IV.

The interelectrode spacing and the thickness of the Si diaphragm are the most critical dimensional parameters of the design. The interelectrode spacing, the fill-gas, and the interior pressure of the chamber determine the discharge initiation (breakdown) voltage. The breakdown voltage between plane-parallel electrodes is given by the Paschen curve (Fig. 2) [20]:

$$
V_{b}=\frac{B p d}{\ln A p d-\ln \left[\ln \left(1+1 / \gamma_{s e}\right)\right]} .
$$

Here $p$ is the fill-gas pressure; $d$ is the effective length of the breakdown path, approximated by the spacing between the electrodes. $A\left(\mathrm{~cm}^{-1} \mathrm{Torr}^{-1}\right)$ and $B\left(\mathrm{~V}-\mathrm{cm}^{-1} \mathrm{Torr}^{-1}\right)$ are parameters obtained by fitting the first Townsend coefficient, $\alpha\left(\mathrm{cm}^{-1}\right)$ as a function of $E / p$ (Electric field/gas pressure), $\alpha=A p \cdot \exp (-(E / p) / B p)$. $A$ and $B$ depend on the type of gas

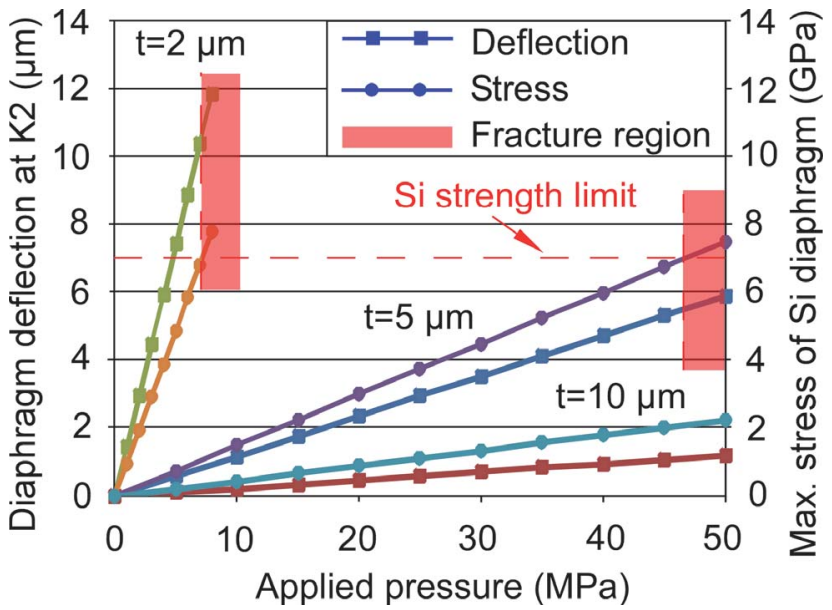

Fig. 3. Simulation results of diaphragm deflection at cathode 2 and maximum induced stress for a $2 \mu \mathrm{m}, 5 \mu \mathrm{m}$, and $10 \mu \mathrm{m}$ thick Si diaphragms.

but not on the metal of the electrodes. The parameter $\gamma_{s e}$ is the secondary electron emission coefficient by ion bombardment, which depends on the metal selected for the electrodes and the gas. A larger $\gamma_{s e}$ lowers the breakdown voltage, which is one of the reasons that $\mathrm{Ni}$ is used for the electrodes. For each choice of fill-gas in the microdischarge chamber, there is a $p \cdot d$ product that corresponds to a minimum voltage for discharge initiation. Operating the device near the minimum of Paschen's curve also has the additional benefit that the energy dissipated by the discharge is reduced. For this work Ar at $1 \mathrm{~atm}$. is chosen for the fill-gas in the microdischarge chamber, because it offers lower operating voltages compared to nitrogen and is cost-effective. Based on this choice of gas and pressure, the $A K 1$ spacing is selected as $10 \mu \mathrm{m}$. The $A K 2$ spacing can be tailored by adjusting the thickness of the bond ring; it is set at $10 \mu \mathrm{m}$ and $30 \mu \mathrm{m}$ in the plasma model and at $30 \mu \mathrm{m}$ in the experiments.

The thickness of Si diaphragm has a significant influence on both sensitivity and dynamic range. A thinner diaphragm offers a high sensitivity but limits the pressure. To determine the appropriate design choice, FEA is performed using $\mathrm{COMSOL}^{\circledR}$ for pressures up to $50 \mathrm{MPa}$ (Fig. 3). A $5 \mu \mathrm{m}$ thick diaphragm supports a large pressure dynamic range while allowing significant deflection. The deflection of the diaphragm supporting the $K 2$ electrode is up to about $5 \mu \mathrm{m}$ $(0.12 \mu \mathrm{m} / \mathrm{MPa})$ within the fracture limit, which is a significant fraction of the initial $A K 2$ spacing.

It should be noted that even for the smallest nominal $A K 2$ gap $(10 \mu \mathrm{m})$, a full scale diaphragm deflection of $5 \mu \mathrm{m}$ will only change the pressure within the microdischarge chamber from $1 \mathrm{~atm}$. to about $1.2 \mathrm{~atm}$. This is provided by the following formulae [21], assuming that the ideal gas law is applicable and the gas inside the chamber is isothermal:

$$
\begin{aligned}
\Delta d & =\frac{3 \cdot \Delta P\left(1-v^{2}\right) a^{4}}{16 E h^{3}} \\
\Delta V & =\frac{\pi \cdot a^{2} \cdot \Delta d}{3}
\end{aligned}
$$

where $\Delta d$ is the deflection at the center of a circular diaphragm, $\Delta P$ is the pressure difference across the 
diaphragm, $a$ is the radius, $h$ is the thickness, $v$ is Poisson's ratio of the material, $E$ is Young's modulus and $\Delta V$ is the volume change due to deflection.

The exterior dimensions of the device are shown in Fig. 1. The total volume of the sensor in the design is $0.05 \mathrm{~mm}^{3}$, whereas the microdischarge chamber is only $2.2 \times 10^{-4} \mathrm{~mm}^{3}$

\section{Microdischarge Modeling}

First principles computer modeling of the microdischarge pressure sensor was performed to provide insights to the physical processes occurring in such devices and to provide guidance in scaling the devices to smaller dimensions. This effort benefited from the availability of a customized 2-dimensional multi-fluid hydrodynamic computational platform, nonPDPSIM developed and refined over a number of years by three of the co-authors [22], [23]. It solves transport equations for all charged and neutral species in the plasma coincident with Poisson's equation for the electric potential and radiation transport. The fundamental equations for charged species are:

$$
\begin{aligned}
& \nabla(\varepsilon \nabla \Phi)=-\left(\sum_{j} q_{j} N_{j}+\rho_{s}\right) \\
& \frac{\partial N_{j}}{\partial t}=-\nabla \cdot \vec{\Gamma}_{j}+S_{j} \\
& \frac{\partial \rho_{s}}{\partial t}=\left[\sum_{j} q_{j}\left(-\nabla \vec{\Gamma}_{j}+S_{j}\right)-\nabla(\sigma(-\nabla \Phi))\right]
\end{aligned}
$$

where $\varepsilon, \Phi, \rho_{s}$ and $\sigma$ are the permittivity, electric potential, surface charge density, and conductivity of solid materials; and for species $j$, the terms $N_{j}, \vec{\Gamma}_{j}, S_{j}$, and $q_{j}$ represent charged species number density, species flux, source function, and charge, respectively. Poisson's equation [Eq. (4)], the transport equations for conservation of the charged species $j$ [Eq. (5)], and the material and surface charge balance equation [Eq. (6)] are simultaneously integrated using a sparse-matrix and Newton iteration technique. Poisson's and charge density equations are solved throughout the computational domain to address electric field penetration into dielectrics. Electrically floating metal features are approximated as dielectrics having sufficiently high conductivities such that the floating feature is equipotential, and there is essentially no internal electric field within it. The electron energy equation is integrated for average energy $E_{\text {ave }}$,

$$
\begin{array}{r}
\frac{\partial\left(n_{e} E_{\text {ave }}\right)}{\partial t}=\vec{j} \cdot \vec{E}-\nabla \cdot\left(\frac{5}{2} \vec{\Gamma}_{e} E_{\text {ave }}-\lambda \nabla T_{e}\right) \\
-n_{e} \sum_{i} \Delta E_{i} \kappa_{i} N_{i}
\end{array}
$$

where $T_{e}$ is the electron temperature defined as $\left(2 E_{\text {ave }} / 3\right)$, $n_{e}$ is the electron density, $\kappa_{i}$ is the rate coefficient for collision process $i$ with species having density $N_{i}$ and energy loss $\Delta E_{i}$, $\lambda$ is the electron thermal conductivity, and $\vec{\Gamma}_{e}$ is the electron flux. Transport and rate coefficients are obtained by solving Boltzmann's equation for the electron energy distribution, and constructing a table of coefficients as a function of $T_{e}$. This table is then interpolated and updated during execution

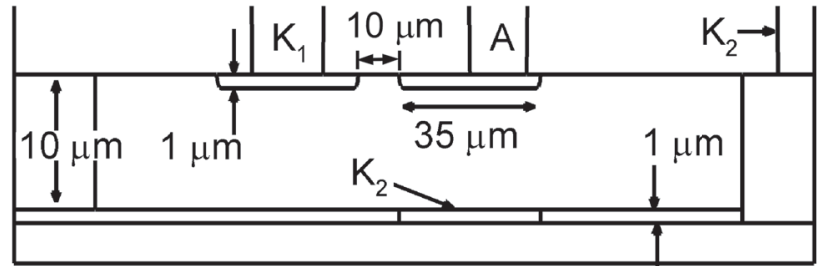

(a)

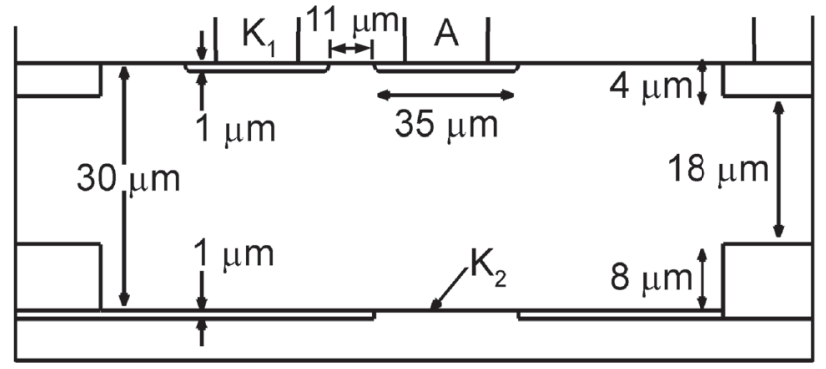

(b)

Fig. 4. Schematics of the two geometries of the microdischarge pressure sensor modeled in this effort. (a) The microdischarge chamber for a fully assembled sensor having a $10 \mu \mathrm{m}$ gap. The microdischarge chamber has the vertical scale expanded by a factor of 3.3 for clarity. (b) The tested device with spacers. The vertical scale is expanded by a factor of 2 .

of the model. These electrons are referred to as bulk electrons. Secondary electrons emitted from surfaces and which are accelerated in the sheaths adjacent to surfaces are referred to as beam electrons. These electrons are tracked using a Monte Carlo simulation. Secondary electrons are emitted from surfaces by bombardment by fluxes of ions and UV photons.

The computational platform, nonPDPSIM uses an unstructured mesh which enables fine features of the actual devices to be modeled over a dynamic range of $10^{4}$, and so an assessment of narrowly spaced elements and small features can be made. The finest resolution of the mesh for cases discussed here is $0.6-0.8 \mu \mathrm{m}$. Although the mesh is static - that is, it does not evolve during the calculation - the change in differential current as a function of pressure can be modeled with a series of calculations, each with a different deformation of the diaphragm. Extensive databases are available for plasma and neutral reactions occurring in the Ar-filled microdischarge chamber [22].

Schematics of the two geometries of the microdischarge pressure sensor modeled here are in Fig. 4. The microdischarge chamber is shown in Fig. 4(a) for a sensor having a $10 \mu \mathrm{m}$ gap, with the vertical scale expanded by a factor of 3.3 for clarity. The glass has a dielectric constant of $\varepsilon / \varepsilon_{0}=3.9$. The reference cathode $(K l)$ and the anode $(A)$ are exposed to the microdischarge chamber with electrodes $1 \mu \mathrm{m}$ in thickness and $35 \mu \mathrm{m}$ wide, separated by $10 \mu \mathrm{m}$. The exposed area of the sensing cathode $(K 2)$ is directly under the anode; elsewhere is it covered with dielectric. This configuration represents the fully assembled device. The device used for experimental validation is shown in Fig. 4(b) (with the vertical scale expanded by a factor of 2).

The simulated electron density and ionization source by bulk and secondary beam electrons for the device with the 




(a)

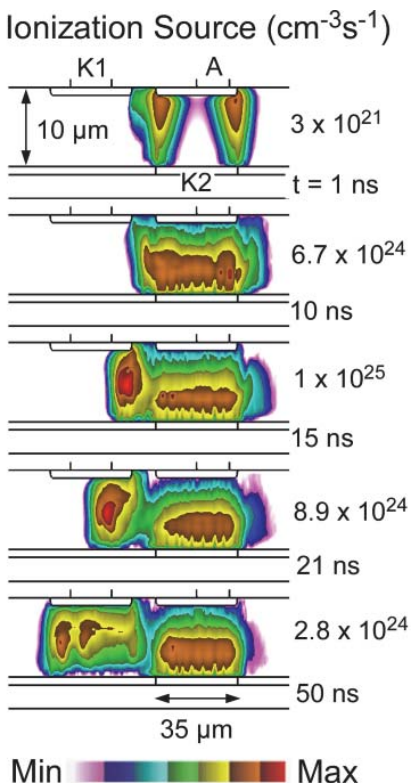

(b)
Fig. 5. Plasma properties for the microdischarge sensor having a $10 \mu \mathrm{m}$ gap operating in $1 \mathrm{~atm}$. of Ar with $400 \mathrm{~V}$ applied to the anode as a function of time after application of voltage. (a) Electron density and (b) ionization source (the sum of ionization by bulk and secondary beam electrons). The flood contours are on a log scale over 4 decades. The maximum value in each frame is noted. (The vertical scale is expanded by a factor of 3.3).

$10 \mu \mathrm{m}$ gap are shown in Fig. 5. The ionization source is the volumetric rate at which electrons and ions are produced by collisions between electrons and atoms. The fill-gas is $1 \mathrm{~atm}$. (760 Torr) of Ar. For this simulation, the applied voltage on the anode is $400 \mathrm{~V}$, while the cathodes are grounded. Ballast resistors of $100 \Omega, 100 \Omega$ and $500 \Omega$ are assumed, in series with $K 1, K 2$, and $A$, respectively. The plasma is initiated by a small amount of electric field emission of electrons from the edges of $K 1$ and $K 2$ resulting in a negligibly small current density of $10^{-2} \mathrm{~A}-\mathrm{cm}^{-2}$. These electrons rapidly avalanche in the geometrically enhanced electric fields at the edges of the anode. The electron density increases from $10^{9}$ to nearly $10^{16} \mathrm{~cm}^{-3}$ over a period of $10 \mathrm{~ns}$, creating a conductive plasma in front of the anode. This conductive plasma then reduces the electric field around the anode, translating the large electric field to the periphery of the plasma and toward the cathodes. When the plasma reaches $K 2$, ion and photon bombardment of its surface produces secondary electrons, which are accelerated back into the plasma and maintain a large rate of ionization. The large electric field directed towards $K 1$ enables the plasma to spread in that direction, eventually covering $K 1$ after about 20 ns. Bulk electrons are prevented from reaching $K 1$ and $K 2$ by the electrodes' large negative potential with respect to the plasma, as shown by the gaps in bulk electron density adjacent to $K 1$ and $K 2$ in Fig. 5(a). Current to these electrodes is provided by the conduction current of ions and displacement current.

The simulated electron density and ionization source in the device with a $30 \mu \mathrm{m}$ gap are shown in Fig. 6. Ballast resistors of $1000 \Omega, 1000 \Omega$ and $20 \mathrm{M} \Omega$ are assumed, in series with



(a)

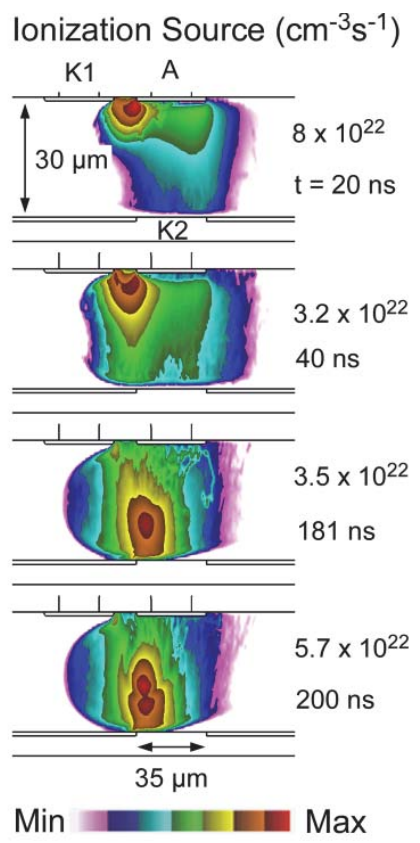

(b)
Fig. 6. Plasma properties for the tested microdischarge sensor having a $30 \mu \mathrm{m}$ gap operating at 770 Torr of Ar with $480 \mathrm{~V}$ applied to the anode as a function of time after application of voltage. (a) Electron density and (b) ionization source (the sum of ionization by bulk and secondary beam electrons). The flood contours are on a log scale over 5 decades. The maximum value in each frame is noted. The vertical scale is expanded by a factor of 2 .

$K 1, K 2$, and $A$, respectively. The pressure is 770 Torr (consistent with the experiments) and the initiating current density from the cathodes is $10^{-4} \mathrm{~A}-\mathrm{cm}^{-2}$. The voltage on the anode is assumed to be $480 \mathrm{~V}$ with a rise time of $5 \mathrm{~ns}$. Although the trends are similar to the smaller $A K 2$ gap device, there are qualitative differences due to the significantly larger gap and ballast resistor. The closer proximity of cathode $K 1$ to the anode $A$ initiates the plasma first between these two electrodes. The larger gap produces a lower electric field between $K 2$ and anode $A$, and so the relative contribution of electric field enhancement to ionization at the edges of the electrodes is greater. The larger ballast resistor produces a larger decrease in voltage across the plasma, since as current is collected there is a larger voltage drop across the ballast resistor. This reduced voltage, combined with the larger gap, produces a lower electric field in the bulk plasma and a lower electron density. A peak value of $10^{14} \mathrm{~cm}^{-3}$ is observed compared to nearly $10^{16} \mathrm{~cm}^{-3}$ for the other compact device.

\section{FABRICATION}

The fabrication process requires 6 masks, 3 for glass processing (Fig. 7) and 3 for silicon processing (Fig. 8). The glass processing includes the laser-drilling of the TGVs, followed by the filling of the vias using copper electroplating. The next steps include the patterning of the contact pads on the exterior side of the wafer and the indium bond ring on the interior side of the discharge chamber. Finally, the Ni electrodes are patterned inside the microdischarge chamber. The silicon processing includes the deposition and patterning 


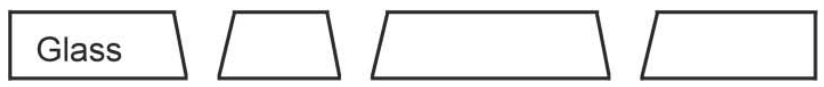

1. Laser drill through glass holes

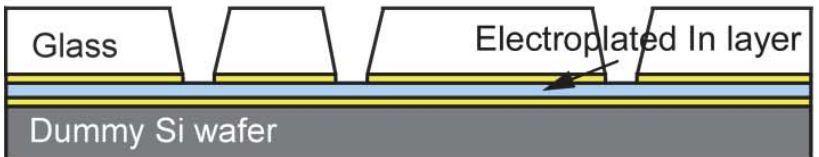

2. Glass and dummy Si wafers Au-In eutectic bond



Dummy Si wafer

\section{Cu electroplating}



4. Lapping from both glass sides



5. Lift-off metal contact pads

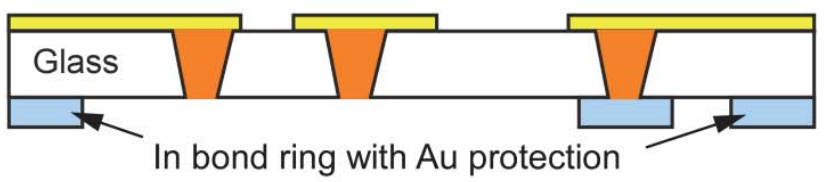

\section{Lift-off In bond ring and K2 contact}

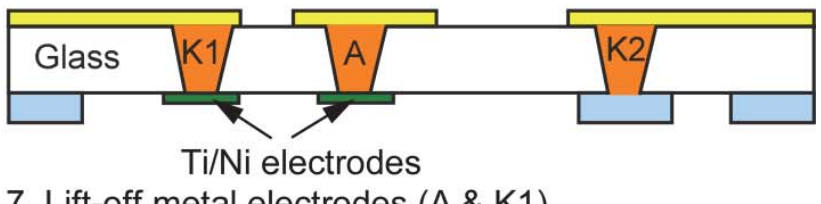

\section{Lift-off metal electrodes (A \& K1)}

Fig. 7. Process sequence for the glass wafer. 1) Through-holes are laser drilled. 2) Glass wafer is attached to a dummy Si wafer by eutectic bonding. A 4-6 $\mu \mathrm{m}$ thick electroplated indium layer on the dummy wafer serves as the seed layer for $\mathrm{Cu}$ electroplating. 3) Through-holes are filled by $\mathrm{Cu}$ electroplating. 4) A lapping step removes excess $\mathrm{Cu}$ and the dummy wafer. 5) The contact pads are deposited and patterned on the exterior of the glass wafer. 6) The bond ring and $K 2$ contact are defined on the interior side of the glass wafer. The In bond ring is covered with $50 \mathrm{~nm} \mathrm{Au}$ for protection. 7) Electrodes are deposited on the interior of the glass wafer.

of an insulating oxide on the $\mathrm{Si}$ device layer of a siliconon-insulator (SOI) wafer. This is followed by the patterning of the $\mathrm{Au}$ bond ring and $K 2$ electrode. Next, the glass and silicon chips are aligned and attached using a Au-In eutectic bonding method. Post-bonding, the Si diaphragm is released from the handle wafer by a deep reactive ion etching (DRIE) process, using the buried oxide layer as the etch stop.

\section{A. Glass Processing}

The glass processing uses $300 \mu$ m-thick Schott Borofloat ${ }^{\circledR}$ glass wafers. In order to provide electrical contact from the

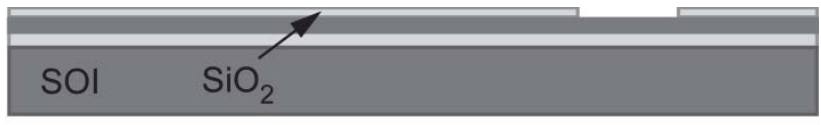

1. Grow silicon dioxide for diaphragm insulation, pattern oxide for $\mathrm{K} 2$ contact to $\mathrm{Si}$



2. Electroplate Au bond ring, lift-off Ti/Ni electrode K2

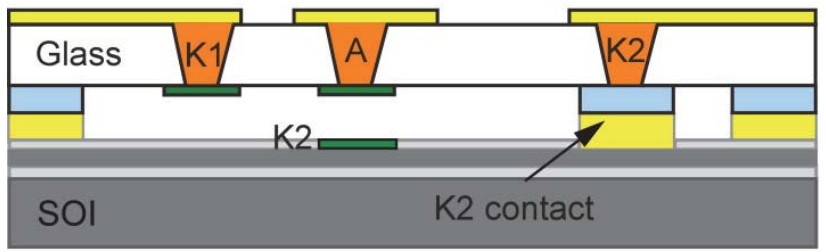

3. Au-In eutectic bond

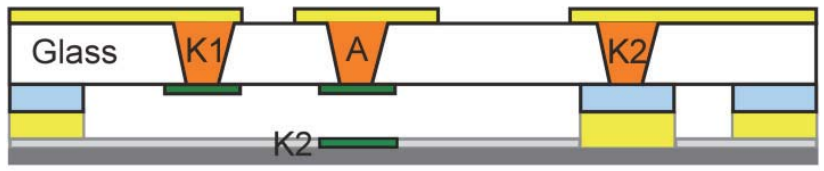

\section{Release handle wafer}

Fig. 8. Process sequence for the $\mathrm{Si}$ wafer. 1) Silicon dioxide layer is grown and patterned on SOI for diaphragm insulation, and $K 2$ contact is then defined. 2) $\mathrm{Au}$ bond ring and $K 2$ contact on the interior of SOI wafer are electroplated. $K 2$ electrode is deposited by a lift-off process. 3) Eutectic bonding is performed between glass and silicon wafers. 4) Handle wafer is released by a backside dry etching.

pressure sensor electrodes (located within the sealed chamber) to the contact pads (located outside the chamber), vias are drilled (Precision Microfab, Severna Park, MD) using a $193 \mathrm{~nm}$ ArF excimer laser. This machining process has a depth control of approximately $\pm 5 \mu \mathrm{m}$, a lateral precision of $1-2 \mu \mathrm{m}$, and a profile taper of $88.1^{\circ}$. The actual machined holes are $47.5 \mu \mathrm{m}$ on the exterior side and $15.8 \mu \mathrm{m}$ on the interior side for a machining profile of $87^{\circ}$ [Fig. 9(a)].

A variety of methods can be used for achieving an electrical connection through the glass vias, including thin-film deposition, packing and melting of solder balls or powder, and electroplating. The high aspect ratios of the TGV structures make it impractical to achieve sufficient sidewall coverage for reliable electrical contacts using thin film deposition. The use of solder particles yields limited success because of inconsistent reflow when heated to the melting temperature $\left(183^{\circ} \mathrm{C}\right.$ for $\left.37 \mathrm{~Pb} / 63 \mathrm{Sn}\right)$ and beyond (up to $\left.280^{\circ} \mathrm{C}\right)$. Although the exact cause of this behavior has not been determined, it is possibly related to the large ratio of surface area to volume, which is known to prevent recrystallization.

Electroplating provides the consistency and scalability for filling the TGVs. Although a variety of plating metals are available, $\mathrm{In}$ and $\mathrm{Cu}$ are the best candidates for this application. Indium has a low reflow temperature $\left(156^{\circ} \mathrm{C}\right)$, which allows temperature cycling post-plating in order to remove pinholes or voids. Copper offers lower resistivity and a higher plating rate. The higher re-melting temperature can also accommodate 


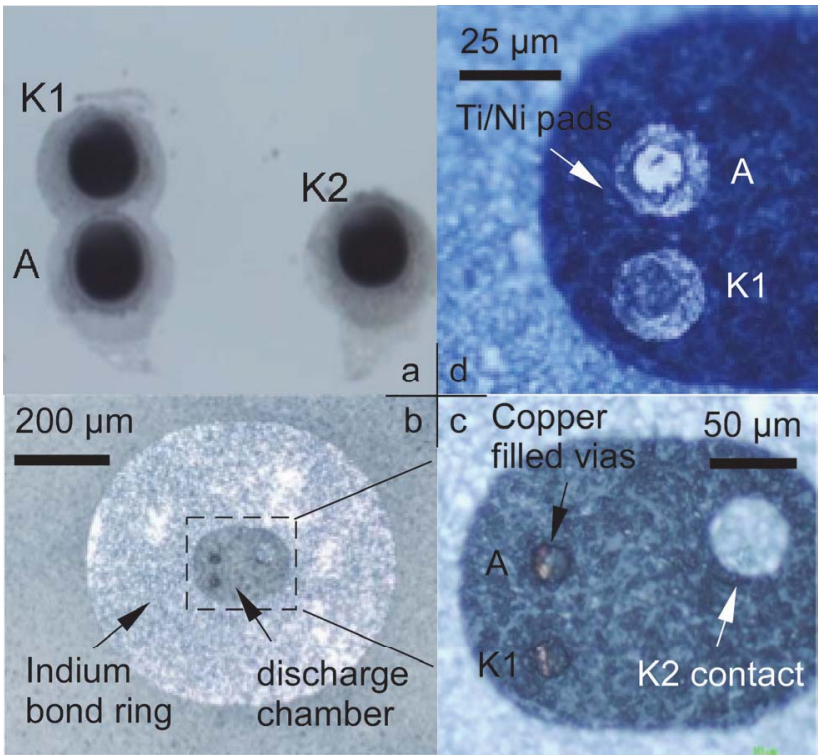

Fig. 9. Optical photos of fabricated glass chip. (a) Laser drilled TGVs, out-chamber side. (b) TGVs are electroplated with copper; bond ring and $K 2$ contact are patterned with indium and Au protection. (c) Zoom-in view of the microdischarge chamber. (d) Anode and cathode 1 are patterned with $\mathrm{Ti} / \mathrm{Ni}$ as electrodes by a lift-off process.

a higher operating temperature for the pressure sensor. Both metals were successfully plated in experiments.

Before electroplating, the glass wafer is attached to a dummy $\mathrm{Si}$ wafer coated with the appropriate metal seed layer (e.g. $\mathrm{Ti} / \mathrm{Au}$ ) for electroplating. Maintaining close contact and minimizing movement between the glass wafer and seed layer are critical. To ensure this, first the glass wafer is coated with a thin layer of $\mathrm{Ti} / \mathrm{Au} 30 \mathrm{~nm} / 300 \mathrm{~nm}$ whereas the dummy $\mathrm{Si}$ wafer is coated with a $30 \mathrm{~nm} / 300 \mathrm{~nm} \mathrm{Ti} / \mathrm{Au}$ layer and a 4-6 $\mu$ m-thick electroplated In layer. Then the dummy Si wafer is bonded to the glass wafer using Au-In eutectic bonding. (Two other attachment options include photoresist to affix the dummy wafer [18] or electroless plating [19].) Following a degassing step to remove bubbles from the vias, $\mathrm{Cu}$ plating is performed (Enthone Cuprostar ${ }^{\circledR} \mathrm{CVF} 1$ ) at $24^{\circ} \mathrm{C}$. Pulse plating with periodic reversal of polarity is used to provide uniform plating across the TGVs on the wafer. The effective current density is $15-20 \mathrm{~mA} / \mathrm{cm}^{2}$. After the plating, the stacked structure is lapped from the front to remove excessive metal build-up and planarize the surface, and from the back to grind off the dummy Si wafer. The measured resistance of the TGV is $<5 \Omega$.

The next processing steps involve patterning the metal contact pads on the exterior side, followed by the patterning of the indium bond ring and Ti/Ni electrodes $(A$ and $K l)$ on the interior of the microdischarge chamber.

The $\mathrm{Ti} / \mathrm{Au}(30 \mathrm{~nm} / 300 \mathrm{~nm})$ contact pads (located on the exterior of the glass chip) are patterned using the lift-off technique. To prevent discharges outside the chamber (i.e., across the contact pad features), the corresponding contacts for the anode and two cathodes are strategically spaced farther apart compared to their spacing inside the chamber $(10 \mu \mathrm{m})$, and partially coated with an insulating layer of epoxy above the TGVs (Fig. 1).
The In bond ring ( $4 \mu \mathrm{m}$-thick, coated with a $50 \mathrm{~nm}$-thick $\mathrm{Au}$ layer) and one side of $K 2$ contact are then formed by evaporation and lift-off (Fig. 9). The Ti/Ni $(20 \mathrm{~nm} / 200 \mathrm{~nm}$ ) electrodes (34.5 $\mu \mathrm{m}$-wide) are formed by lift-off achieving an AK1 spacing of approximately $11.1 \mu \mathrm{m}$. As shown in Fig. 9(d), the alignment of the TGVs and $\mathrm{Ni}$ electrodes is reasonably good and centered.

\section{B. Silicon Processing}

The silicon processing utilizes SOI wafers with a Si device layer (5 $\mu \mathrm{m}$-thick), a buried silicon dioxide layer ( $2 \mu \mathrm{m}$-thick), and a Si handle wafer (500 $\mu$ m-thick). The Si device layer includes As doping for low resistivity $(<0.005 \Omega-\mathrm{cm})$; this is necessary to electrically route the $K 2$ electrode to the $K 2$ contact. When the glass and $\mathrm{Si}$ wafers are bonded, the $K 2$ contact on the $\mathrm{Si}$ wafer electrically connects to the $K 2$ contact on the glass wafer. This connection is then routed through the TGV to the contact pad for $K 2$. The buried oxide layer provides a well-defined etch stop, which can later facilitate the final diaphragm release of the Si device layer by a backside dry etch of the handle wafer.

Silicon dioxide is grown $(100 \mathrm{~nm}$-thick, by dry oxidation at $1000^{\circ} \mathrm{C}$ ) and then deposited $(900 \mathrm{~nm}$-thick low temperature oxide) for a total thickness of $1 \mu \mathrm{m}$ on the Si device layer to provide electrical isolation of the bond ring from the $K 2$ electrode and contact. The oxide is patterned using a dry etch process based on $\mathrm{CHF}_{3}$ and $\mathrm{CF}_{4}$ to expose the doped device layer for the $K 2$ contact. Next, an $8 \mu$ m-thick $\mathrm{Au}$ bond ring and $K 2$ contact are provided by electroplating. In a following step, the oxide is removed in the region of the $K 2$ electrode, which is then formed by sputtering and lift-off of $\mathrm{Ti} / \mathrm{Ni}(20 \mathrm{~nm} / 200 \mathrm{~nm})$.

\section{Eutectic Bonding}

The transient liquid phase bonding technique has been used for vacuum packaging [24], [25] and wafer level attachment of ceramics (e.g., PZT) to Si [26]. The motivations include: (1) a relatively low initial melting temperature $\left(200^{\circ} \mathrm{C}\right)$ for the bonding step with the potential for high re-melting temperatures e.g., $500^{\circ} \mathrm{C}$ with $\mathrm{Au}-\mathrm{In}$; (2) the ability to bond a variety of surface profiles; and (3) the ability to control the thickness of the bond layer. The Au-In system was first investigated as a fluxless soldering technique in electrical packaging [27], [28]. There are two basic types of Au-In bonding systems depending on the percent weight of the two metals: indium-rich [27] and gold-rich bonding [28]. If the weight percentage of indium is higher than 54\%, also called "indium-rich", the alloy is a mixture of indium and $\mathrm{AuIn}_{2}$, which means the re-melting (or de-bonding) temperature is still $156^{\circ} \mathrm{C}$. If the weight percentage of indium is between 36.8-54 wt.\%, the alloy is a mixture of AuIn and AuIn 2 intermetallic compounds with a re-melting temperature of $495.4^{\circ} \mathrm{C}$ and is considered "gold-rich".

For the pressure sensor described in this paper, Au-rich bonding is used to ensure a relatively high operating temperature $\left(\approx 500^{\circ} \mathrm{C}\right)$. This bonding process does not depend on 


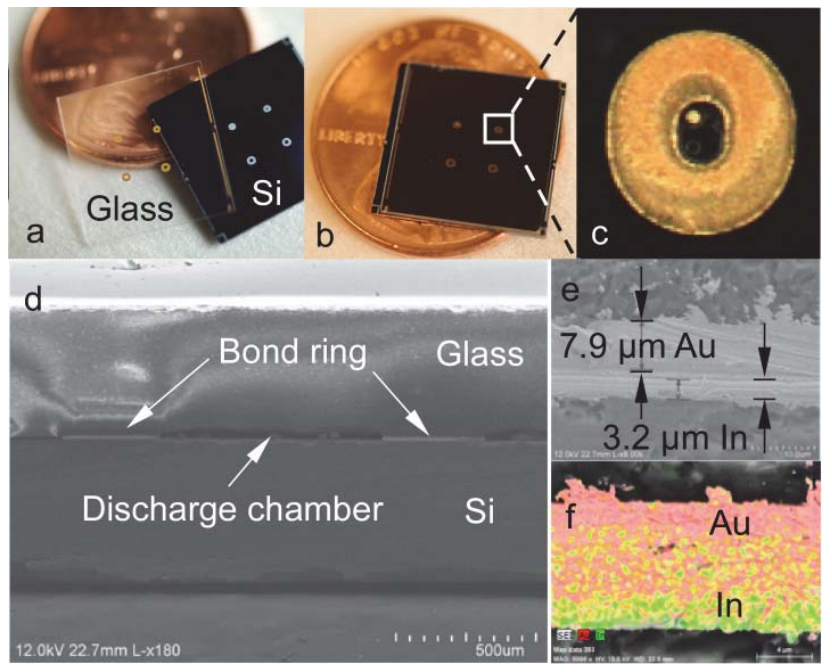

Fig. 10. Chip level eutectic bonding of Au-In bond rings. (a) Glass and silicon chips, before and (b) after bonding, against a U.S. penny (Ø19.05 mm). (c) Zoom-in view of one bonded ring, viewed through glass. (d) SEM image of the cross-section of the Au-In bond ring. (e) SEM details of the bond structure. (f) Electron dispersive spectroscopy shows the diffusion of the $\mathrm{Au}$ and In components and the resulting $\mathrm{Au}$ and In intermetallic compounds that have formed.

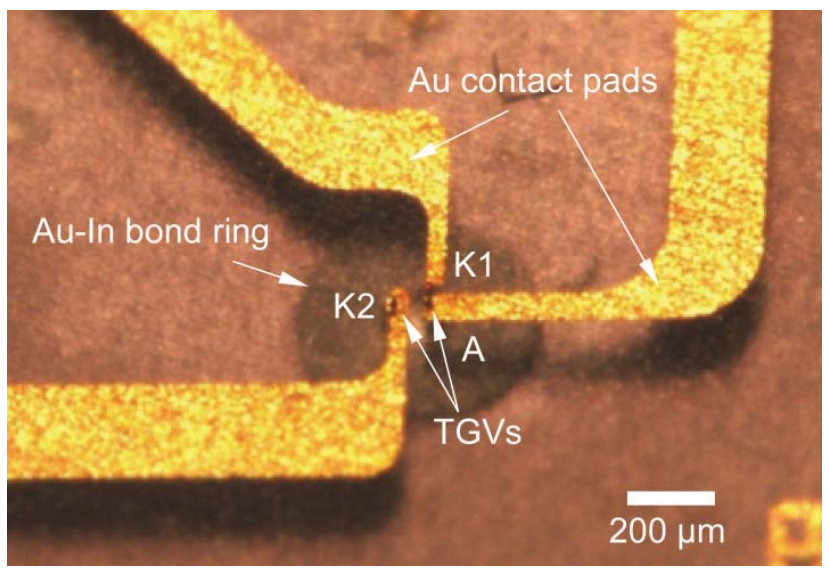

Fig. 11. Photograph of the final assembly of the glass and silicon chips, viewed from the exterior of the glass.

substrate materials (either glass or silicon). In this experimental investigation both cases, with $\mathrm{Au}$ on glass/In on silicon, and $\mathrm{Au}$ on silicon/In on glass were studied and successfully bonded. The bonding was performed in a vacuum oven at $200^{\circ} \mathrm{C}$ with an applied pressure $>1 \mathrm{MPa}$ for $90-120$ mins. Fig. 10(d) shows the cross-section of the bond ring structure captured using scanning electron microscopy (SEM). Electron dispersive spectroscopy (EDS) was used to evaluate the composition of the bond ring [Fig. 10(f)]. The EDS shows diffusion of the Au and In layers that form the intermetallic compounds.

\section{EXPERIMENTAL RESULTS}

To evaluate the impact of multiple conditions for the interior of the microdischarge chamber, a test structure was formed in which the SOI wafer was thinned to $100 \mu \mathrm{m}$, but the handle wafer was not completely removed (Fig. 11). The electrical characterization was performed with a glass chip

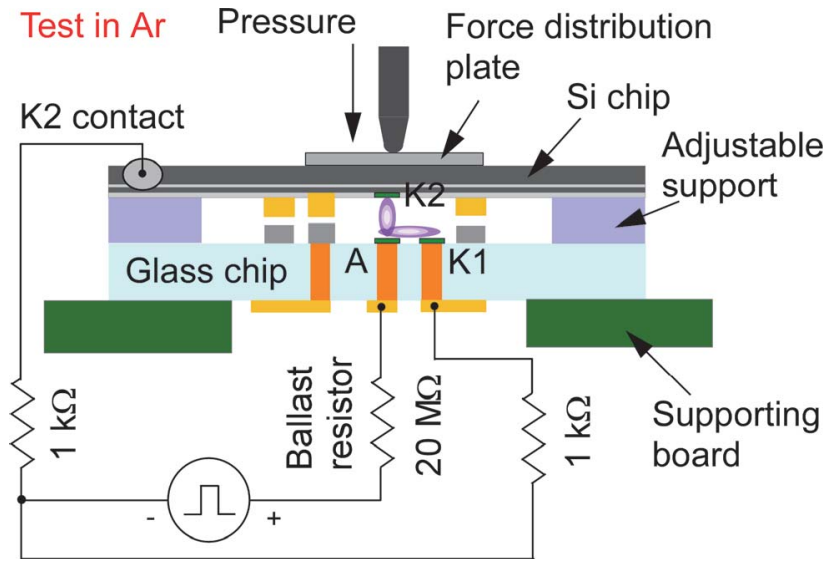

Fig. 12. Test setup for the glass and Si chip assembly. A micromanipulator applies force at the center of the $100 \mu \mathrm{m}$-thick Si diaphragm to deflect the Si chip and change the $A K 2$ spacing.

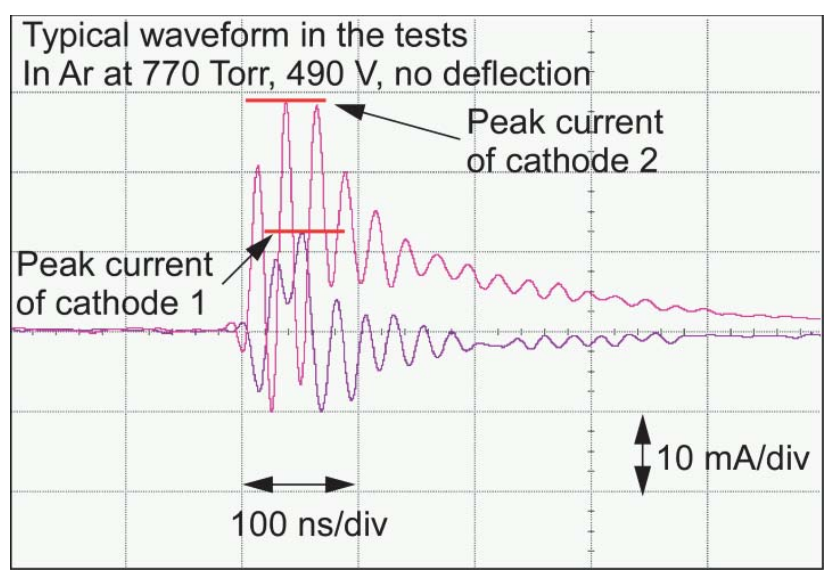

Fig. 13. Representative waveforms of the microdischarges collected at the cathodes obtained from an oscilloscope during the tests.

and a SOI chip that were held together with a porous epoxy bond instead of an eutectic bond. The test structure was left unsealed and tested in an argon environment. The experimental setup is illustrated in Fig. 12. A piezoelectric actuator was used to apply a force at the center of the assembled chip to induce a diaphragm deflection, which emulates a large external pressure. Voltage pulses of $1 \mathrm{~ms}$ duration were applied to the anode. Multiple microdischarge pulses were produced during each voltage pulse. Ballast resistor values of $10 \mathrm{M} \Omega$ and $20 \mathrm{M} \Omega$ were utilized in separate sets of experiments, while the currents going through two competing cathodes $I I$ and $I 2$, were captured as voltages across $1 \mathrm{k} \Omega$ resistors.

A representative waveform of a single microdischarge pulse is shown in Fig. 13. The typical duration is several hundred nanoseconds with decaying oscillation. Possible sources of parasitic capacitance, to which the oscillation may be attributed, include the oscilloscope probes connected to $K 1$ and $K 2$. When a voltage pulse is applied to the anode, it also charges the parasitic capacitance on the anode, which can potentially contribute to the peak transient discharge currents.

The relation between differential current and estimated equivalent pressure is plotted in Fig. 14. The force applied 




Fig. 14. Differential currents from test results versus estimated equivalent pressure and inter-electrode gap from simulation results based on a $5 \mu \mathrm{m}$ thick Si diaphragm. $I 1$ and $I 2$ are peak values of envelope curves for $A K 1$ and $A K 2$ discharge waveforms, respectively. Tests are in Ar with the microdischarge chamber interior pressure between 650 Torr and 770 Torr. Applied voltage pulses are $480 \mathrm{~V}$ to $580 \mathrm{~V}$. Ballast resistors are $10 \mathrm{M} \Omega$ and $20 \mathrm{M} \Omega$. Every point is the average of 5-8 measurements. Error bars indicate one standard error.

to the diaphragm was converted to equivalent pressure using FEA. In four sets of experiments, the impacts of chamber pressure, applied voltage, and ballast resistor were investigated. When the ballast resistor value increases, the nature of the microdischarges changes and impacts the distribution of the cathode currents. For a chamber pressure of 770 Torr and using a $20 \mathrm{M} \Omega$ ballast resistor, the operating voltage was $480 \mathrm{~V}$ and the differential currents ranged from -0.35 to -0.5 . With a smaller $10 \mathrm{M} \Omega$ ballast resistor, the differential currents uniformly decreased.

Although the device is intended to operate with the microdischarge chamber at about 1 atm., the impact of lower pressure was also evaluated (Fig. 14) with the test structure. At an interior pressure of 650 Torr, the (fractional) current in $K 1$ was lowered, as expected from the increase in the mean free path. Additionally, the operating voltage increased from $480 \mathrm{~V}$ for 770 Torr to $580 \mathrm{~V}$ for 650 Torr; this was also expected, given the nature of the Paschen curve for Ar.

The impact of operating voltage on the discharge characteristics is also evident in Fig. 14. For the microdischarge chamber pressure of 770 Torr and a ballast resistor of $10 \mathrm{M} \Omega$, two magnitudes of voltage pulses were evaluated: $480 \mathrm{~V}$ and $500 \mathrm{~V}$. For the $480 \mathrm{~V}$ pulses, the resulting differential current distribution as a function of diaphragm deflection does not indicate a clear trend. However, at $500 \mathrm{~V}$, the impact of relative deflection can be clearly observed in Fig. 14. This indicates the existence of a minimum threshold for the operating voltage of the pressure sensor. Thus, the combination of voltage $(480 \mathrm{~V})$ and ballast resistor $(10 \mathrm{M} \Omega)$ is not expected to be selected for use.

Based on the electrical results, the mechanical load changed $A K 2$ by approximately $5 \mu \mathrm{m}$. This corresponds to the deflection expected from a $5 \mu \mathrm{m}$ thick diaphragm of oval shape under an external pressure of about $40 \mathrm{MPa}$ as noted in Section II B, and in Fig. 3.

\section{DISCUSSION}

The plasma modeling in this work provides insights to the fundamental processes responsible for the differential current, including plasma initiation, evolving and current sustaining. This simulation guides the electrodes design and device scaling to smaller dimension, although no quantitative data directly predict and match experimental results which indeed are affected by multiple experimental conditions.

The device is interrogated using high voltage pulses, and the energy consumption for each pulse is about $5 \mathrm{~mJ}$. For previously reported work on pulsed microdischarge-based devices [8], the energy consumption can be as low as 2-20 $\mu \mathrm{J} /$ pulse. The power consumption for device described in this work depends on the duty cycle, but for 1 reading each second, it is $\sim 5 \mathrm{~mW}$.

Looking forward, it is notable that devices incorporating microdischarges are attractive for high temperature operation as electron temperatures are typically many $\mathrm{eV}$ $(1 \mathrm{eV}=11,600 \mathrm{~K})$ and so are not significantly perturbed by a high ambient temperature [29], [30]. For the conditions encountered in these devices, ions have temperatures moderately above ambient with transport coefficients that are also not particularly sensitive to high operating temperatures. Microdischarge-based pressure sensors have been operated as high as $1000^{\circ} \mathrm{C}$ [3]. Other pressure sensors for high temperature utilize Fabry-Perot and other interferometers [1], and piezoresistors in high band gap materials such as $\mathrm{SiC}$ (up to $600^{\circ} \mathrm{C}$ ) [31] and even $\mathrm{Si}$ (up to $600^{\circ} \mathrm{C}$ ) [32]. The temperature tolerance of monolithic microdischarge-based pressure sensors will be investigated in our future efforts.

\section{CONCLUSION}

There are a number of conclusions that can be drawn from the effort described. First, the differential cathodes arrangement that was investigated - with anode $(A)$ and reference cathode $(K l)$ on the glass substrate, and the sensing cathode (K2) on the diaphragm - was demonstrated to produce a differential output current that was a function of diaphragm deflection. For an $A K 1$ spacing of $10 \mu \mathrm{m}$, the fractional differential current changed by approximately $20 \%$ as $A K 2$ changed from $30 \mu \mathrm{m}$ to $25 \mu \mathrm{m}$. The overall device size was $0.585 \times 0.54 \times 0.2 \mathrm{~mm}^{3}$. It was demonstrated that microdischarges could be initiated at voltages below $500 \mathrm{~V}$ in an Ar filled microdischarge chamber at about 1 atm. interior pressure. Further, the peak current levels were at a level of $\approx 10 \mathrm{~mA}$, and pulse durations were $\approx 100 \mathrm{~ns}$, which permits the discharge to remain relatively energy efficient. First principles computer modeling confirmed the roles of the two cathodes in the operation of the device. The microdischarge is initiated in the $A K 1$ gap and travels to $A K 2$. The two cathodes then compete for current. The modeling also shows that the device can operate over a wide range of $A K 2$, from $10 \mu \mathrm{m}$ to $30 \mu \mathrm{m}$. Tightly packed, high aspect ratio TGVs can be fabricated by combining laser drilling with electroplating. The electroplating can be performed with the aid of a Si dummy wafer that supports a seed layer; the wafer is eutectically bonded to the underside of the glass substrate, and later removed by lapping. 
Although the device design and fabrication approaches may continue to evolve, differential microdischarge currents present a viable approach to sensing diaphragm deflection, and so can be implemented in a variety of devices, such as gas or liquid pressure sensors in harsh environments.

\section{ACKNOWLEDGMENT}

The authors would like to thank David Chapman and the advisory committee members of AEC for their support and encouragement. Facilities used for this research include the Lurie Nanofabrication Facility (LNF), the University of Michigan, Ann Arbor.

\section{REFERENCES}

[1] Y. B. Gianchandani, C. Wilson, and J.-S. Park, "Micromachined pressure sensors: Devices, interface circuits, and performance limits," in The MEMS Handbook, M. Gad-el-Hak, Ed. Cleveland, OH, USA: CRC Press, 2006, pp. 3.1-3.44.

[2] S. A. Wright and Y. B. Gianchandani, "Controlling pressure in microsystem packages by on-chip microdischarges between thin-film titanium electrodes," J. Vac. Sci., Technol. B, vol. 25, pp. 1711-1720, Sep./Oct. 2007.

[3] S. A. Wright and Y. B. Gianchandani, "Discharge-based pressure sensors for high-temperature applications using three-dimensional and planar microstructures," J. Microelectromech. Syst., vol. 18, pp. 736-743, Jun. 2009.

[4] S. A. Wright, H. Z. Harvey, and Y. B. Gianchandani, "A microdischargebased deflecting-cathode pressure sensor in a ceramic package," J. Microelectromechan. Syst., vol. 22, no. 1, pp. 80-86, 2013.

[5] R. Foest, M. Schmidt, and K. Becker, "Microplasmas, an emerging field of low-temperature plasma science and technology," Int. J. Mass Spectrom., vol. 248, pp. 87-102, Feb. 2006.

[6] C. G. Wilson and Y. B. Gianchandani, "Spectral detection of metal contaminants in water using an on-chip microglow discharge," IEEE Trans. Electron Devices, vol. 49, no. 12, pp. 2317-2322, Dec. 2002.

[7] V. Karanassios, "Microplasmas for chemical analysis: Analytical tools or research toys," Spectrochim. Acta Part B, Atomic Spectrosc., vol. 59, pp. 909-928, Jul. 2004.

[8] B. Mitra and Y. B. Gianchandani, "The detection of chemical vapors in air using optical emission spectroscopy of pulsed microdischarges from two- and three-electrode microstructures," IEEE Sensors J., vol. 8, no. 8, pp. 1445-1454, Jul./Aug. 2008.

[9] C. K. Eun and Y. B. Gianchandani, "Microdischarge-based sensors and actuators for portable microsystems: Selected examples," IEEE J. Quantum Electron., vol. 48, no. 6, pp. 814-826, Jun. 2012.

[10] K. Ishii, Y. Hirano, and Y. Murakami, "Gap length-dependent excited Xe-atom density distribution in superfine-pitch discharge cells for coplanar-structure plasma display panels," IEEE Trans. Plasma Sci., vol. 36, no. 4, pp. 1192-1193, Aug. 2008.

[11] S. J. Park, K. F. Chen, N. P. Ostrom, and J. G. Eden, "40,000 pixel arrays of ac-excited silicon microcavity plasma devices," Appl. Phys. Lett., vol. 86, p. 111501, Mar. 2005.

[12] Y. B. Gianchandani, S. A. Wright, C. K. Eun, C. G. Wilson, and B. Mitra, "Exploring microdischarges for portable sensing applications," Anal. Bioanal. Chem., vol. 395, pp. 559-575, Oct. 2009.

[13] X. Luo, C. K. Eun, and Y. B. Gianchandani, "Fabrication of a monolithic microdischarge-based pressure sensor for harsh environments," in Proc. IEEE/ASME Int. Conf. MEMS, San Francisco, CA, USA, Jan. 2014 pp. 64-67.

[14] C. S. Song, Z. Y. Wang, Q. W. Chen, J. Cai, and L. T. Liu, "High aspect ratio copper through-silicon-vias for 3D integration," Microelectron. Eng., vol. 85, pp. 1952-1956, Oct. 2008.

[15] N. T. Nguyen, E. Boellaard, N. P. Pham, V. G. Kutchoukov, G. Craciun, and P. M. Sarro, "Through-wafer copper electroplating for three-dimensional interconnects," J. Micromech. Microeng., vol. 12, pp. 395-399, Jul. 2002.

[16] C. D. Gu, H. Xu, and T. Y. Zhang, "Fabrication of high aspect ratio through-wafer copper interconnects by reverse pulse electroplating," J. Micromech. Microeng., vol. 19, p. 065011, Jun. 2009.

[17] R. Gueye, T. Akiyama, D. Briand, and N. F. de Rooij, "Fabrication and formation of $\mathrm{Ta} / \mathrm{Pt}-\mathrm{Si}$ ohmic contacts applied to high-temperature through silicon vias (TSVs)," Sens. Actuators A, Phys., vol. 191, pp. 45-50, Mar. 2013.
[18] X. G. Li, T. Abe, Y. X. Liu, and M. Esashi, "Fabrication of high-density electrical feed-throughs by deep-reactive-ion etching of Pyrex glass," J. Microelectromech. Syst., vol. 11, pp. 625-630, Dec. 2002.

[19] V. Sukumaran, T. Bandyopadhyay, V. Sundaram, and R. Tummala, "Low-cost thin glass interposers as a superior alternative to silicon and organic interposers for packaging of 3-D ICs," IEEE Trans. Compon. Packag. Manuf. Technol., vol. 2, no. 9, pp. 1426-1433, Sep. 2012.

[20] M. A. Lieberman and A. J. Lichtenberg, Principles of Plasma Discharges and Materials Processing, 2nd ed. Hoboken, NJ, USA: Wiley, 2005.

[21] M. Gad-el-Hak, The MEMS Handbook. New York, NY, USA: Taylor \& Francis, 2006.

[22] B. Lay, R. S. Moss, S. Rauf, and M. J. Kushner, "Breakdown processes in metal halide lamps," Plasma Sources Sci., Technol., vol. 12, pp. 8-21, Feb. 2003.

[23] Z. M. Xiong, E. Robert, V. Sarron, J. M. Pouvesle, and M. J. Kushner, "Dynamics of ionization wave splitting and merging of atmosphericpressure plasmas in branched dielectric tubes and channels," J. Phys. D. Appl. Phys., vol. 45, p. 275201, Jul. 2012.

[24] Y. C. Sohn et al., "Wafer-level low temperature bonding with Au-In system," in Proc. 57th Electron. Compon. Technol. Conf., 2007, pp. 633-637.

[25] R. Straessle, Y. Petremand, D. Briand, and N. F. de Rooij, "Evaluation of thin film indium bonding at wafer level," Proc. Eurosens. Eng., vol. 25 no. 5, pp. 1493-1496, 2011.

[26] E. E. Aktakka, H. Kim, and K. Najafi, "Wafer level fabrication of high performance MEMS using bonded and thinned bulk piezoelectric substrates," in Proc. IEEE Int. Conf. Solid-State Sens., Actuators, Microsyst. (Transducers), Jun. 2009, pp. 849-852.

[27] W. W. So and C. C. Lee, "Fluxless process of fabricating In-Au joints on copper substrates," IEEE Trans. Compon. Packag. Technol., vol. 23, no. 2, pp. 377-382, Jun. 2000.

[28] C. C. Lee, C. Y. Wang, and G. Matijasevic, "Au-in bonding below the eutectic temperature," IEEE Trans. Compon. Hybrids, Manuf. Technol., vol. 16, no. 3, pp. 311-316, May 1993.

[29] M. J. Kushner, "Modelling of microdischarge devices: Plasma and gas dynamics," J. Phys. D, Appl. Phys., vol. 38, pp. 1633-1643, Jun. 2005.

[30] C. G. Wilson, Y. B. Gianchandani, R. R. Arslanbekov, V. Kolobov, and A. E. Wendt, "Profiling and modeling of dc nitrogen microplasmas," J. Appl. Phys., vol. 94, pp. 2845-2851, Sep. 2003

[31] A. Ned, R. Okojie, and A. Kurtz, "6H-SiC pressure sensor operation at $600{ }^{\circ} \mathrm{C}$," in Proc. Int. High Temp. Electron. Conf., Albuquerque, NM, USA, 1998 , pp. 257-260.

[32] S. Guo, H. Eriksen, K. Childress, A. Fink, and M. Hoffman, "High temperature high accuracy piezoresistive pressure sensor based on smartcut SoI," in Proc. IEEE Int. Conf. Micro Electro Mech. Syst., Tucson, AZ, USA, Jun. 2008, pp. 892-895.

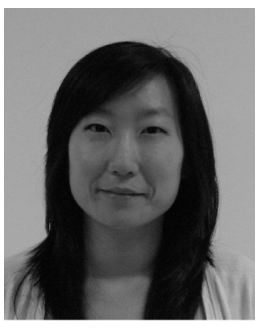

Christine Kay Eun received the B.S. (summa cum laude), M.S., and Ph.D. degrees in electrical engineering from the University of Michigan, Ann Arbor, in 2004, 2006, and 2011, respectively. She has been funded through various fellowships including the Rackham Merit Fellowship and a fellowship from Sandia National Laboratories, Albuquerque, NM.

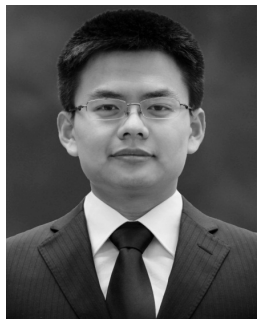

Xin Luo received the B.S. degree in Automotive Engineering from Tsinghua University, Beijing, China, in 2010. He is currently working as a Graduate Student Research Assistant towards the Ph.D. degree in the Department of Mechanical Engineering, University of Michigan, Ann Arbor, with a focus on MicroElectroMechanical Systems. His research interests include microsensors for harsh environments. 


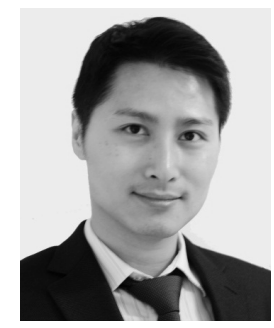

Jun-Chieh Wang is a Ph.D. student in the Department of Electrical Engineering and Computer Science at the University of Michigan (UM), Ann Arbor, MI, USA. He received his B.S. and M.S. in physics from National Cheng Kung University, Tainan, Taiwan, in 2003 and 2005, respectively. Before UM, he worked on theoretical and computational plasma physics in the areas of fusion plasma and ionosphere plasma phenomenon. Since 2009 he has been pursuing a Ph.D. at the University of Michigan in Prof. Mark Kushner's group with a research focus on low temperature plasma science and technology.

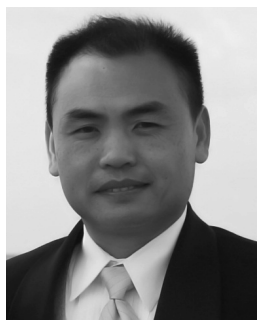

Zhongmin Xiong was a research scientist at University of Michigan whose research focuses on numerical modeling of fluid flow, gas discharge and low temperature plasmas. Prior to joining University of Michigan, he worked as a simulation and modeling engineer for research and product development in Schlumberger Ltd. from 2007 to 2009. He did his post-doctoral research on numerical simulations of tokamak edge plasmas at Lawrence Livermore National Lab from 2004 to 2007. He obtained his $\mathrm{Ph} . \mathrm{D}$. in flow physics and computation from Stan-

ford University in 2004.
Mark Kushner, photograph and biography not available at the time of publication.

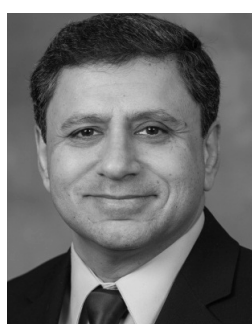

Yogesh Gianchandani (S'83-M'85-SM'05-F'10) is a Professor at the University of Michigan, Ann Arbor, with a primary appointment in the Electrical Engineering and Computer Science Department, and a courtesy appointment in the Mechanical Engineering Department. He also serves as the Director for the Center for Wireless Integrated MicroSensing and Systems $\left(\right.$ WIMS $^{2}$ ).

Dr. Gianchandani's research interests include all aspects of design, fabrication, and packaging of micromachined sensors and actuators (http://www.eecs.umich.edu/ yogesh/). He has published about 300 papers in journals and conferences, and has about 35 US patents issued or pending. He was a Chief Co-Editor of Comprehensive Microsystems: Fundamentals, Technology, and Applications, published in 2008. Dr. Gianchandani has served on the editorial boards and program committees of a number of conferences and journals. From 2007 to 2009 he also served at the National Science Foundation, as the program director for Micro and Nano Systems within the Electrical, Communication, and Cyber Systems Division (ECCS). 